\title{
Novel c.2216T $>$ C (p.I739T) Mutation in Exon 13 and c.1481T > A (p.L494X) Mutation in Exon 8 of MUT Gene in a Female with Methylmalonic Acidemia
}

\author{
George Imataka - Osamu Sakamoto · Hideo Yamanouchi • \\ Shigemi Yoshihara $\cdot$ Yuki Omura-Hasegawa · \\ Go Tajima $\cdot$ Osamu Arisaka
}

Published online: 12 March 2013

(C) The Author(s) 2013. This article is published with open access at Springerlink.com

\begin{abstract}
We report herein a 1.5-year-old girl with methylmalonic acidemia (MMA) in whom two missense mutations were found: a novel I739T mutation located in exon 13 and the L494X mutation in exon 8. The results of organic acid test showed a pronounced increase in methylmalonate excretion with increased methylcitrate and 3-OH-propionate excretion, leading to a diagnosis of MMA, and Vitamin B12 administration was started. Analysis of the mut gene confirmed a T-to-A substitution at nucleotide position 1481 in exon 8 and a T-to-C substitution at nucleotide position 2216 in exon 13, leading to the amino acid isoleucine at position 739 being changed to threonine, resulting in c.2216T $>\mathrm{C}$ (p.I739T). The patient
\end{abstract}

G. Imataka $(\bowtie) \cdot S$. Yoshihara $\cdot$ O. Arisaka

Department of Pediatrics, School of Medicine, Dokkyo Medical

University, 880 Kitakobayashi, Mibu, Shimotsuga,

Tochigi 321-0293, Japan

e-mail: geo@dokkyomed.ac.jp

O. Sakamoto

Department of Pediatrics, Tohoku University School

of Medicine, Sendai, Miyagi, Japan

H. Yamanouchi

Department of Pediatrics, Saitama Medical University,

Saitama, Japan

Y. Omura-Hasegawa

Department of Pediatrics, Shimane University Faculty of

Medicine, Izumo, Shimane, Japan

G. Tajima

Department of Pediatrics, Graduate School of Biomedical \& Health Sciences, Hiroshima University, Hiroshima, Japan has now been on high-dose oral administration of Vitamin B12 and carnitine therapy ( $900 \mathrm{mg}$ of levocarnitine chloride) for 5 years without experiencing further attacks, and her cognitive and motor development is normal. Further tests on residual enzyme activity, as well as experience with more cases, may shed light on the relationship between gene mutations and phenotypes in MMA.

Keywords Methylmalonic acidemia .

L-Methylmalonyl-CoA mutase · Vitamin B12

\section{Introduction}

Methylmalonic acidemia (MMA; MIM\# 251000) is an autosomal recessively inherited disorder in which a defect in methylmalonyl CoA mutase (MCM, EC5.4.99.2) results in the build-up of methylmalonic acid and other organic acids in the body. MMA is categorized as either apoenzyme deficiency (mut) type or coenzyme B12 metabolic disorder $(\mathrm{Cbl})$ type. The $m u t$ type is subdivided into $m u t^{0}$, which results from complete deficiency, and $\mathrm{mut}^{-}$, which results from partial deficiency. The $m u t^{0}$ type, which is characterized by apoenzyme activity of not more than $0.1 \%$, often presents with metabolic acidosis within 1 week after birth, frequently resulting in death in early childhood. The $m u t^{-}$type presents with repeated attacks of ketoacidosis triggered by infection after the age of 1 year. MCM is encoded by a single gene, mut, which is located on $6 \mathrm{p} 21$. Mut consists of 13 exons [1-3]. We herein report a case of a girl with MMA in which two missense mutations were found: a novel I739T mutation located in the most C-terminal exon 13 ever reported and the L494X mutation located in exon 8, which is relatively common in Japanese patients. 


\section{Patient Report}

A healthy 1.5-year-old girl went to sleep one night after vomiting. In the morning, the parents found the girl unconscious in bed and took her to the emergency room. The girl was $76.3-\mathrm{cm}$ tall and weighed $8.4 \mathrm{~kg}$ with a body temperature of $35.2{ }^{\circ} \mathrm{C}$ and blood pressure of $77 / 47 \mathrm{mmHg}$. She did not open her eyes in response to speech, and the results of simple blood glucose test were below the detectable limit. She regained consciousness following administration of $20 \%$ blood glucose, but experienced tonic convulsions and rolling eyes for several minutes. The convulsions were controlled with phenobarbital. Intravenous fluids were started. The patient was lucid after $3 \mathrm{~h}$, and her motor functions improved after $6 \mathrm{~h}$ to the point where she could walk on her own. Blood gas analysis at the time the patient was hospitalized showed $\mathrm{pH}$ 7.240, $\mathrm{pCO}_{2}$ $41.5 \mathrm{mmHg}$, base excess -9.2 , and $\mathrm{HCO}_{3}-17.2 \mathrm{mmol}$, indicating metabolic acidosis. Other abnormal results were lactic acid, $35 \mathrm{mg} / \mathrm{dL} ; \mathrm{NH}_{3}, 118 \mu \mathrm{g} / \mathrm{dL}$; AST, $143 \mathrm{U} / \mathrm{L}$; and ALT, $46 \mathrm{U} / \mathrm{L}$. Urine specific gravity was 1.04 with a urine ketone level of $3+$.

Organic acid test was performed on suspicion of congenital metabolic anomaly. The results showed a pronounced increase in methylmalonate excretion with increased methylcitrate and 3-OH-propionate excretion, leading to a diagnosis of MMA. Because the approximate urine methylmalonic acid level at onset was $658.4 \mu \mathrm{M} / \mathrm{mmol}$ creatinine, high-dose oral administration of Vitamin B12 (10 $\mathrm{mg}$ of cobamamide) was started, resulting in a decrease to $213.0 \mu \mathrm{M} / \mathrm{mmol}$ creatinine on day 7 of treatment.

HPLC quantification of succinyl-CoA production upon reaction of the patient's lymphocyte lysates with methylmalonyl-CoA and adenosylcobalamin revealed that the patient's methylmalonyl-CoA activity level was 1.4 pmol succinyl-CoA $/ \mathrm{min} / 10^{6}$ cells, which is approximately $5 \%$ of the level seen in healthy controls $(35.9 \pm 16.4$ pmol succinyl-CoA $/ \mathrm{min} / 10^{6}$ cells).

The patient has now been on high-dose oral administration of Vitamin B12 and carnitine therapy ( $900 \mathrm{mg}$ of levocarnitine chloride) for 5 years. She has not experienced further attacks; her cognitive and motor development is normal.

\section{mut Genetic Analysis}

Analysis of the mut gene was performed for definitive analysis of the type of MMA in this patient. A T-to-A substitution at nucleotide position 1481 in exon 8 of the mut gene was found in a heterozygous pattern (Fig. 1a).

A T-to-C substitution at nucleotide position 2216 in exon 13 was also found in a heterozygous pattern. As a result of this base substitution, the amino acid isoleucine at position 739 was changed to threonine, resulting in c.2216T $>C$ (p.I739T). The child was therefore definitively diagnosed with methylmalonic acidemia (mut) (Fig. 1b).

\section{Discussion}

In this case of MMA, we found the L494X mutation in exon 8 , which is common in Japanese patients, and an I739T mutation in exon 13, which has never previously been reported. The patient in this case presented with convulsions associated with hypoglycemia. The clinical course after hospitalization was relatively mild for MMA. Blood and urinalysis data suggested that this case of MMA

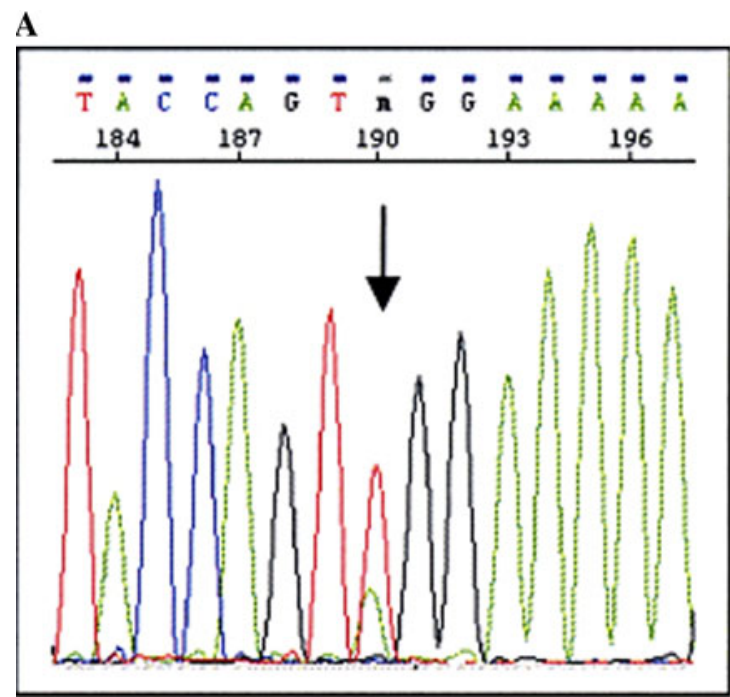

B

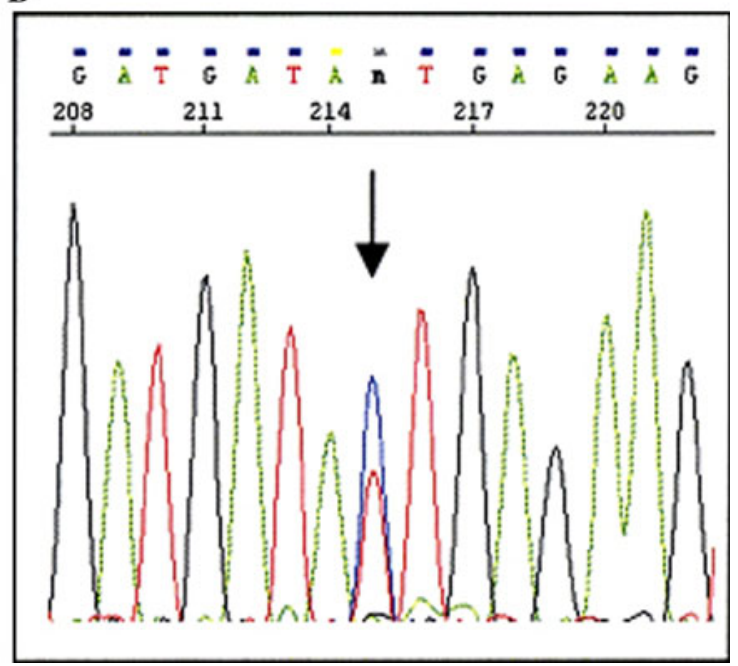

Fig. 1 a Mutation c.1481T > A (p.L494X) in exon 8 of $M U T$ gene. b Novel c.2216T > C (p.I739T) mutation in exon 13 of $M U T$ gene 
was an organic acid metabolic disorder, and the patient was treated with high doses of Vitamin B12 after more detailed analysis. Before the start of Vitamin B12 treatment, the approximate urine methylmalonic acid levels were $658.4 \mu \mathrm{M} / \mathrm{mmol}$ creatinine, as compared with $213.0 \mu \mathrm{M} /$ mmol creatinine after treatment. This case was therefore originally thought to involve Vitamin B12-reactive MMA. However, HPLC quantification of succinyl-CoA production upon reaction of the patient's lymphocyte lysates with methylmalonyl-CoA and adenosylcobalamin revealed that the child's enzyme activity was approximately $5 \%$ of normal, which was not indicative of the $\mathrm{B} 12$ reactive type. We therefore performed mut genetic analysis. Ultimately, a novel I739T mutation in exon 13 and the L494X mutation in exon 8 were found, resulting in a definitive diagnosis of the $m u t^{-}$type MMA.

Of the 119 mutations of the mut gene reported previously, the novel I739T mutation in exon 13 found in this case is the closest to the $\mathrm{C}$ terminal [1-5]. The ( $\beta$, a)5cobalamin (vitamin B12) binding domain occurs in C-terminal exons 10,11, 12, and 13 of the mut gene. An extremely interesting point in this case is that the reaction shown by urine methylmalonic acid levels following clinical administration of Vitamin B12 was consistent with the location of the genetic mutation. This warrants further analysis, including in vitro tests on the expression of residual enzyme activity after the addition of Vitamin B12. Further experience with more cases may shed light on the relationship between gene mutations and phenotypes in MMA.

Open Access This article is distributed under the terms of the Creative Commons Attribution License which permits any use, distribution, and reproduction in any medium, provided the original author(s) and the source are credited.

\section{References}

1. Sakamoto, O., Ohura, T., Matsubara, Y., Takayanagi, M., \& Tsuchiya, S. (2007). Mutation and haplotype analyses of the MUT gene in Japanese patients with methylmalonic academia. Journal of Human Genetics, 52, 48-55.

2. Kobayashi, A., Kakinuma, H., \& Takahashi, H. (2006). Three novel and six common mutations in 11 patients with methylmalonic academia. Pediatrics International, 48, 1-4.

3. Acquaviva, C., Benoist, J.-F., Perwira, S., Callebaut, I., Koskas, T., Porquet, D., et al. (2005). Molecular basis of methylmalonyl CoA mutase apoenzyme defect in 40 European patients affected by mut $^{0}$ and mut ${ }^{-}$forms of methylmalonic academia: Identification of 29 novel mutations in the MUT gene. Human Mutation, 25, 167-176.

4. The Human Gene Mutation Database at the Institute of Medical Genetics in Cardiff. Retrieved Oct 2012 from http://www.hgmd. cf.ac.uk/ac/all.php.

5. Merinero, B., Perez, B., Perez-Cerda, C., Rincon, A., Desviat, L. R., Martinez, M. A., et al. (2008). Methylmalonic acidaemia: Examination of genotype and biomedical data in 32 patients belonging to mut, cb1A or cb1B complementation group. Journal of Inherited Metabolic Disease, 31, 55-66. 